\title{
Natural History of Chronic Pyelonephritic Scarring
}

\author{
C. J. HODSON,* F.R.C.P., F.F.R. ; S. WILSON,* B.SC.
}

Brit. med. F., 1965, 2, 191-194

It may well be that the term "chronic pyelonephritis," as Kleeman et al. (1960) suggest, represents a broad spectrum of chronic renal disease. Certain it is that many topical discussions tend to forsake the classical entity, which is perhaps best known as " atrophic pyelonephritis," and to range widely among all renal conditions that may give rise to recurrent pyuria or bacteriuria. Nevertheless it is this same atrophic pyelonephritis, or small coarsely scarred kidney, which is still responsible for the majority of deaths from uraemia and for a high proportion of cases of hypertension of renal origin. It is with the large scars found in this condition that this contribution is concerned in an endeavour further to support the statements of previous authors, and to focus attention once more on the very early age of onset of this condition in most cases. The method used is simple enough-namely, delineation of the scar by means of intravenous pyelography.

The large irregular scar seen in the natural specimen is nearly always associated with destruction of the related renal pyramid. It differs from the scar due to infarction in that with the latter the associated pyramid remains, albeit reduced in size (Smith, 1962). It is thus easily recognized in excretion pyelography when this procedure is conducted so that the renal outlines are defined (either by careful preliminary preparation of the abdomen or by means of tomography). The scar reveals itself as a localized narrowing of the renal substance, often only a few millimetres thick, which is associated with distortion, blunting, or complete absence of the adjacent pyramid, depending on its size and age. There may or may not be a flattening or depression in the renal outline at the site (Hodson, 1959). In fact, in many cases the renal outline is remarkably smooth. This appearance can be exactly mimicked by chronic ulcerating tuberculosis, by back-pressure atrophy behind a calculus jammed in a calix, and by what has become known as the "phenacetin" kidney, except that in the last instance necrotizing papillitis results in the frequent sloughing and separation of the pyramid, which has not yet been seen in our series of pyelonephritic scars.

When generalized throughout the kidney such scarring is of ten confused with generalized back-pressure atrophy due to prolonged obstruction of urine-flow at the renal pelvis or in the ureter. This also presents as a flattening or recession of the actual pyramids associated with narrowing of the renal substance, but without scarring. Also, these effects are present uniformly and to an equal degree throughout and produce a radiological appearance which is quite different from the very irregular result of renal scarring. The two can be clearly differentiated, except sometimes in the very small contracted kidney. In fact, experience over 10 years, often checked by surgical and necropsy evidence, has shown that radiology provides a very reliable means of diagnosing this scarred form of chronic pyelonephritis during life, and thus a means of studying the life-history of this condition in a manner not possible by other methods of observation.

It must be emphasized that often a scar can be so extensive that it must represent the gross destruction of a tissue mass amounting at times to most of an upper or lower renal pole. The original acute lesions must presumably be of the same considerable magnitude, as there is no evidence to support the local extension of a scar. Little mention has so far been made

* University College Hospital, London. of the large size of these scars, particularly in children. There may well be other variations which are not definable on pyelography, and which may well be multiple and of clinical significance. The present study is confined to the large scars and necessarily does not include such lesions. The part they may play in the overall problem is as yet unknown.

\section{Material and Approach}

The cases which form the material for this study were collected over a period of eight years. They derive from a large teaching hospital in which there is a flourishing paediatric department and from a children's hospital, both of which are situated in the Central London area. The amount of case selection involved in both instances is difficult to define, but probably does not differ greatly from that pertaining to any other urban hospital.

The relationship of the results obtained to the population as a whole must be considered only in the light of these sources of derivation. Nevertheless it is our belief that they represent a not too distorted picture.

The selection of the first group is entirely on a radiological basis and the presence of scarring, and consists of the first 100 cases so diagnosed. The second group, as described by Smellie et al. (1964), consisted of 200 consecutive cases of urinary infection occurring in children under the age of 12 years who attended the teaching hospital.

The approach to the problem which we have adopted is the result of an impression that has grown over the years-namely, that there seemed to be many more children with pyelonephritic scarring than could be accounted for by its incidence in the adult population examined. Adults were, of course, referred for $x$-ray examination for a variety of clinical conditions. Large numbers derived from a flourishing hypertension clinic under the care of Professor Rosenheim; others came because of recurrent urinary infection from gynaecological and general medical and surgical out-patient departments ; the rest presented with the common urinary complaints usual to an adult population. In the eight years under review, on a conservative estimate, from 12,000 to 13,000 adult pyelographic examinations were made. Over the same period perhaps 3,000 such examinations were undertaken in patients under the age of 20 . These are rough figures, and in the last three or four years an

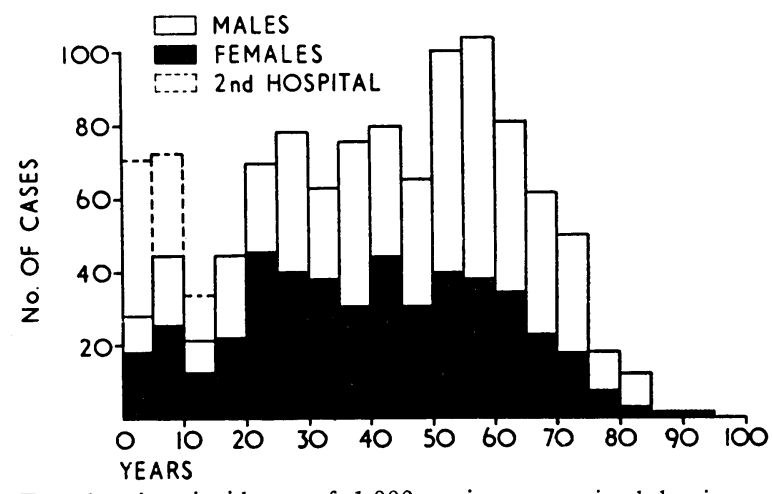

FIG. 1.-Age incidence of 1,000 patients examined by intravenous pyelography in a sample year at both hospitals. 
increasing number of children were included-the age attendance over an average year is given in Fig. 1-but they are sufficient to indicate the samples involved and to outline the background from which our impression was developed.

The logical way to investigate the validity of this impression seemed to be to analyse the whole group of kidneys showing coarse pyelonephritic scarring from the point of view of age incidence at diagnosis, and then to attempt to relate these findings to the population sample examined.

\section{Cases of Pyelonephritic Scarring}

The cases selected were the first 100 we encountered that presented the typical appearance of chronic pyelonephritic scarring as defined above. Where there was any doubt, or where such associated conditions as stone formation or tumour were present, cases were omitted. The age stated represents the age at diagnosis; probably scarring had been present in some cases for years. A few general remarks may be made about the findings.

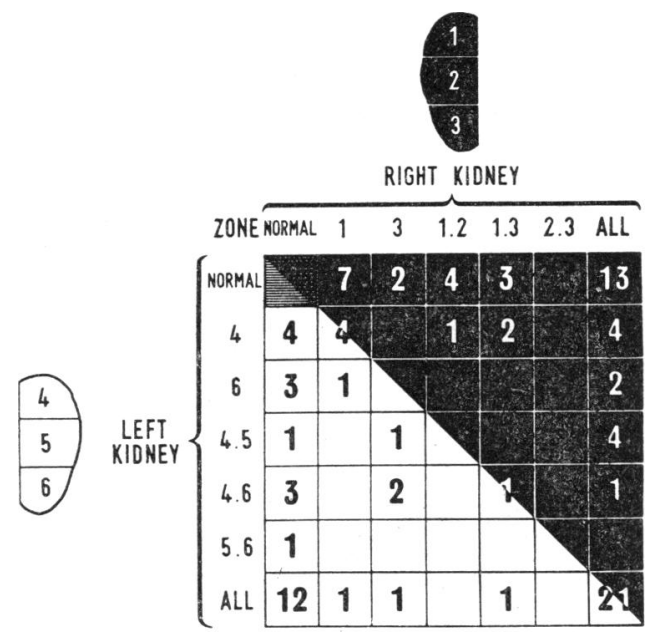

FIG. 2.-Latin scuare showing distribution of scars in 100 cases of chronic pyelonephritic scarring.

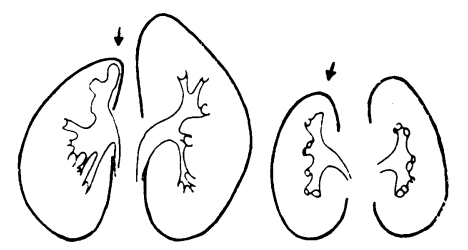

1. UPPER POLE SCARRING

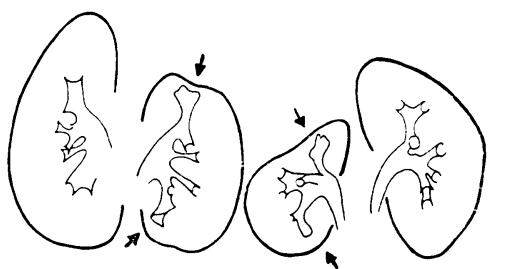

2. UPPER AND LOWER POLE SCARRING

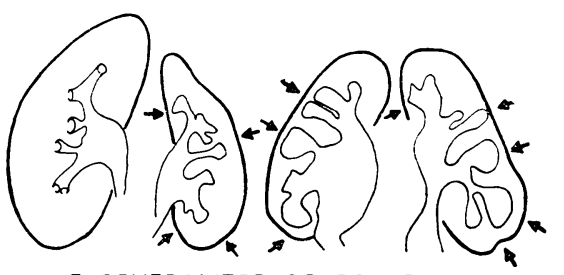

3. GENERALIZED SCARRING

FIG. 3.-Tracings of pyelograms showing (1) unipolar, (2) bipolar, and (3) Eeneralized scarring in unilateral and bilateral disease.
The age of these patients at the time of diagnosis ranged from a few months to 61 years ; 21 were males. The distribution of scars varied from case to case, but certain common patterns of scarring were often found. They are represented as a Latin square in Fig. 2. The right upper pole was most commonly the site of a single scar, followed by the left upper pole and both lower poles. Oblique views of the kidneys were not taken as a routine during these examinations, and it is possible, but improbable, that middle-zone scars occur with greater frequency than here represented. Bipolar scarring was not uncommon. More frequently (in 13 patients on the right side and in 12 on the left) scarring was distributed throughout the whole of one kidney. Most commonly, however, in 21 patients, generalized scarring was present throughout both kidneys. A particularly interesting finding was that total disease of the right kidney was associated with one or more scarred areas in the left in 11 patients, while the opposite occurred in only three. Scarring in all degrees and in all sites is, in fact, more common in the right kidney. Tracings of three grades of scarring are shown in Fig. 3. Scar distribution might well form a rational way of grading renal damage.

In the large majority of patients with unilateral disease the opposite kidney was above average size; in many it was markedly hypertrophied. This was noticeable in children as well as in adults.

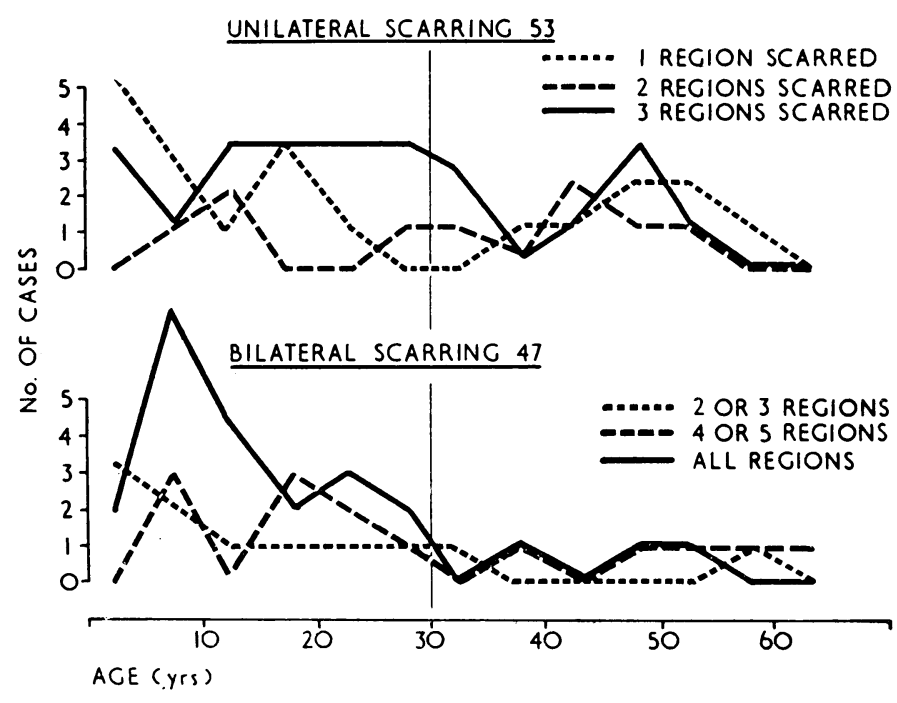

FIG. 4.-Age of 100 patients at time of diagnosis of pyelonephritic scarring.

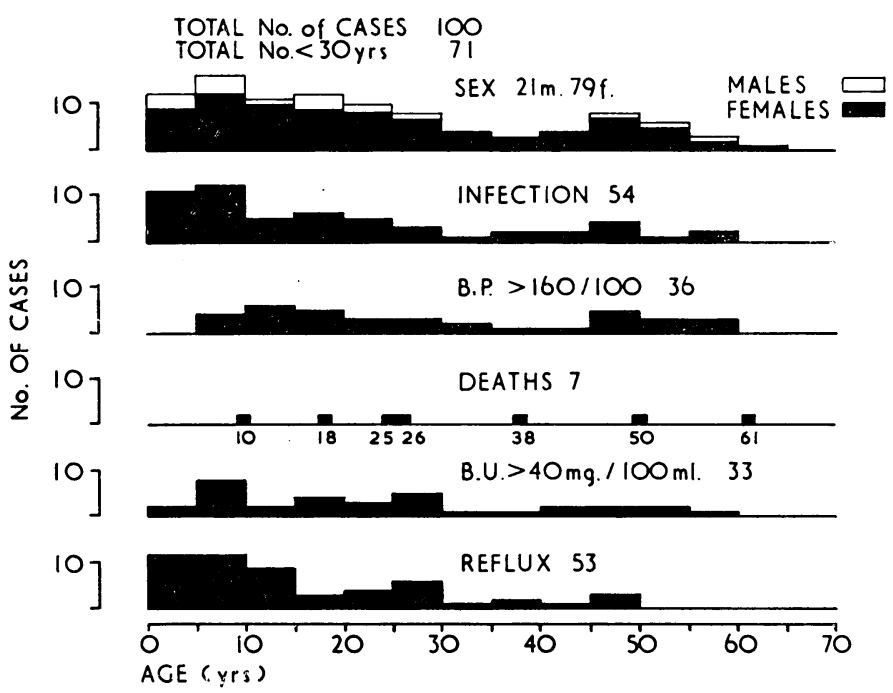

FIG. 5.--Related clinical features which were associated at time of diagnosis, or later during the period of follow-up-namely, hypertension, infection, vesico-ureteric reflux, raised blood urea, and death. (in decades.) 
The time required for a scar to form is still unknown, but severe bipolar scarring developed in one child over a period of 15 months ( $7 \frac{1}{4}$ to $8 \frac{1}{2}$ years).

In Figs. 4 and 5 are set out the breakdown of scarring into early, moderate, and severe, unilateral and bilateral related to age at diagnosis, and the important clinical phenomena that were found then, or later, in these patients.

In Fig. 6 scarring is related to the age incidence of pyelographic examination shown in Fig. 1. This is a more correct representation of actual age incidence.

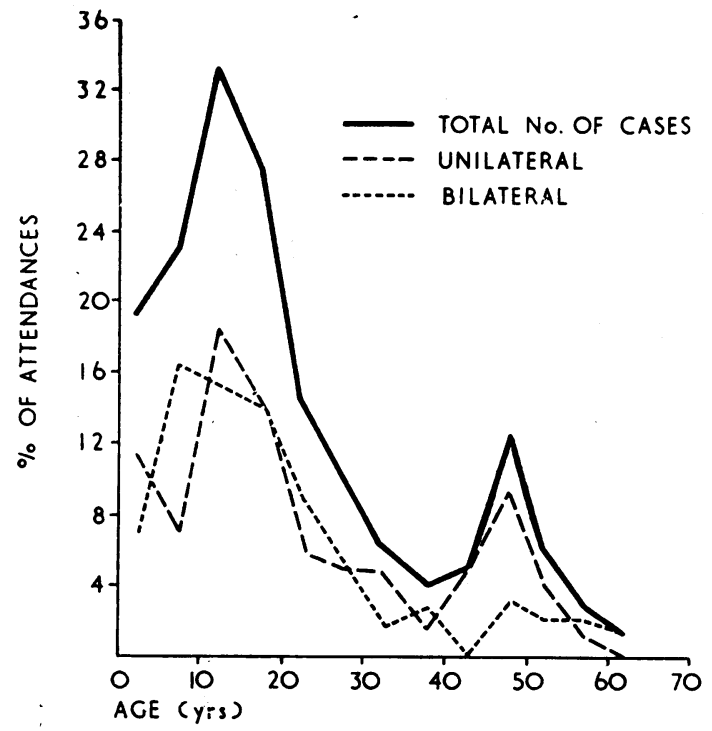
grams performed in the various decades.

Fifty-three patients had either a history of recurrent attacks of fever associated with urinary symptoms (mainly children) or a significant urine culture, and in these infection was regarded as proved. In 35 patients the blood-pressure was persistently raised above $160 / 100 \mathrm{~mm}$. $\mathrm{Hg}$. In 33 the blood urea was persistently above $40 \mathrm{mg} . / 100 \mathrm{ml}$. Vesico-ureteric reflux was demonstrated in 53 cases out of 67 in which a micturating cystogram was performed. Deaths occurred at all ages.

The pattern of scarring shows two interesting features. Severe unilateral scarring decreases sharply in incidence towards the end of the fourth decade, with a further rise between 45 and 55 years. Moderate and advanced bilateral scarring is at maximum incidence about 10 years and falls off rapidly to nil at.the age of 45 , with a further slight rise between the ages of 45 and 60

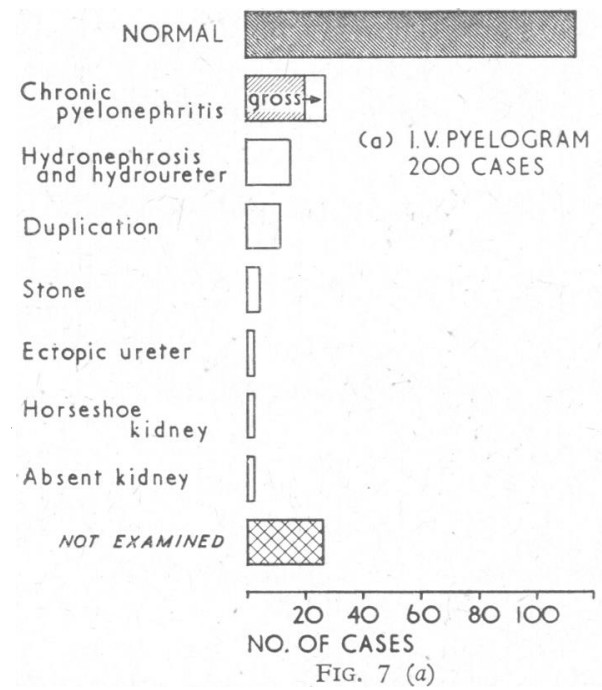

FIG. 6.-Scarring in Fig. 4 related to the number of pyelo-

years (Fig. 6). It is noticeable also that the clinical features of infection, raised blood-pressure, elevated blood urea, and vesicoureteric reflux follow very closely the incidence of bilateral scarring.

The significance of this decline in the incidence of severe scars is, of course, vital. On the surface it would seem to indicate a fatal outcome in these patients, and this is supported by much previous writing and statistical evidence about the disease. However, this conclusion has certain statistical weaknesses that preclude its being completely acceptable. Among other things, it is not known how many such cases, both unilateral and bilateral, may become quiescent, the patients remaining healthy enough not to seek further investigation at a later age. It seems inescapable, however, that pyelonephritic scarring of all grades is very common among children.

In short, the results of this analysis appeared not only to support our original impression but to proffer a dramatic and sinister explanation for it. It was, indeed, the disquieting number of cases of scarring demonstrated in young children which was partially responsible for initiating the review published by Smellie et al. (1964) in an attempt to define the overall picture in this age-group more clearly. The relevant part of this work is now briefly outlined.

\section{Cases of Urinary Infection in Patients Under 12 Years}

Three criteria govern the inclusion of cases in this series: (1) their age, (2) a positive urine culture on one or more occasions, and (3) more than 10 pus cells per high-power field $(1 / 6)$ on one or more occasions. The radiological investigations consisted of excretion pyelography (175 cases) and micturating

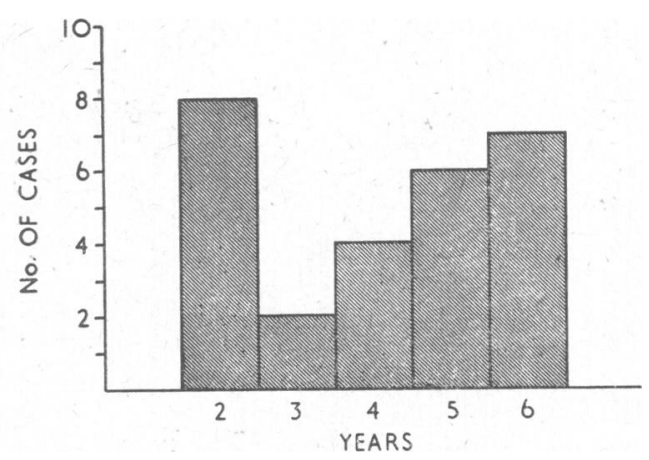

FIG. 8.-Age at diagnosis of the 26 patients with pyelonephritic scarring referred to in Fig. 7.

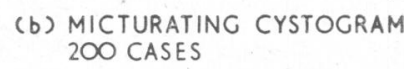

FIG. 7.-Results of routine radiological investigation of 200 consecutive cases of urinary infection in children under 12 years. (a) 175 excretion pyelograms; (b) 165 micturating cysto-urethrograms. Note incidence of pyelonephritic scarring and vesico-ureteric reflux. 
cysto-urethrography (165 cases). The patients were limited to those attending the paediatric department of the teaching hospital referred to above. Thirty-eight came direct from its obstetric unit in the neonatal period.

The results are illustrated in Fig. 7. Of the 175 cases submitted to excretion pyelography, pyelonephritic scarring was found bilaterally in 7 and unilaterally in 19 patients, a total percentage of 15.0. The next most frequent urinary lesion was permanent dilatation of the ureter or renal pelvis, which was about half as frequent as pyelonephritic scarring. Of the 165 micturating cystograms, $56(34 \%)$ showed unilateral and bilateral vesico-ureteric reflux.

All scars found were present by the age of 6 years.

In Fig. 8 the incidence of pyelonephritic scarring is related to age at diagnosis.

\section{Discussion}

Of the various methods by which the course of chronic pyelonephritis may be assessed-clinical, bacteriological, by biopsy, by surgery, or from the accumulated evidence of postmortem examinations-none provides a full picture and all are open to misinterpretation. The radiological assessment, although by no means comprehensive, is at least complementary and allows study of the individual cases.

Moreover, in the growing kidney it is our experience that the rate of its growth is as valuable a guide to health as the rate of growth of the child as a whole. So that a slowing up of kidney growth can be used as a valuable indication of renal disease.

It was the result of this process of serial observations in children with pyelonephritic scarring and vesico-ureteric reflux which originally caused us to hold such a gloomy view of the prognosis of this condition. It was the comparatively few cases found in adults which led, as mentioned before, to the present study. There is no dearth of warnings in the published literature. Escherich himself, as far back as 1897, emphasized the incidence of infection of the urinary tract in children. Weiss and Parker (1939), in their classical description, pointed to the early origin of the disease in many cases. Campbell (1951) found urinary infection in $0.8 \%$ of 47,000 admissions to a children's hospital, with a peak of incidence between 12 and 18 months, regarding it as "probably outranking all other bacterial disease under the age of two." Kunin et al. (1964) in a survey of 8,000 schoolgirls found urinary infection in $1.2 \%$. And several recent authors have drawn attention once again to the difficulty of diagnosis in the very young.

The two series of cases mentioned here indeed only highlight an already established point of view, and show once again that advanced chronic pyelonephritis is the commonest disease of the kidneys in children.

What is brought to the fore, perhaps, is that this lesion, when bilateral, is mortal in the vast majority of cases, leading to death before the age of 35 , often in the second decade. When unilateral it may also be lethal in most cases, when unrecognized and untreated.

What is not yet clear is the origin of those cases, both unilateral and bilateral, which are occasionally found in the older age-groups. They may represent the survivors of a childhood infection which has become quiescent in some instances; in others they may result from infection incurred by women in early marital life or during or after pregnancy. Further study is required to settle this point.

What is suggested is that the majority of this latter adult group of urinary infections do not scar to the same degree as the childhood cases, and are not so commonly associated with vesico-ureteric reflux. This is also supported by the experience of serial pyelographic studies. In children it is the rule, when infection is not permanently controlled by maintenance antibiotics, to find that progressive scar formation occurs in both unilateral and bilateral cases, once scarring has begun. This may occur at any time between the age of 2 and 10 years, and such deterioration is often rapid. In adults, on the other hand, new scar formation is comparatively rare, apart from the effects of neurogenic disease of the bladder. In the series described only three patients showed scarring during the period of observation. In two this followed operations on the lower end of the ureter and was associated with vesico-ureteric reflux. In the third, an advanced bilateral case, it followed a further incident of urinary infection.

It is of course impossible to say how long scarring has been present when the patient is first seen. The size (amount of hypertrophy) of the opposite kidney may be some guide. But it is not unusual for an adult case to remain unchanged, as regards distribution and degree of scarring, for 5 or 10 years; and so it is highly probable that in some adult cases the scarring developed in childhood.

Finally, it is suggested that these findings appear to justify a broad subdivision of chronic pyelonephritis, on gross morbid anatomical grounds, into the coarsely scarred group commonly originating in early childhood, with an extremely bad prognosis, and a second group, perhaps with smaller scars, which arise later in life and to which there is a much more hopeful prognosis. The natural history of coarse pyelonephritic scarring suggested by these observations must lead to an intensification of methods of detection and control of urinary infection in infants. This is likely to be a formidable task because of the vagueness of signs and symptoms and the practical difficulties of obtaining reasonable urine specimens. Until this problem is tackled and an effective answer found that is easily applicable by general practitioners in home conditions, this disease will continue to scourge our innocents, and the sequence of protracted ill-health and irreversible kidney damage leading to early death will as surely follow.

\section{Summary}

Experience over eight years of the radiological diagnosis of chronic pyelonephritic scarring produced a strong impression that this was mainly a disease of children.

Analysis of 100 consecutive cases of such scarring revealed the highest proportion between 10 and 15 years, with a steady decrease up to the age of 45 and a small subsequent rise in later years.

This is construed as indicating a fatal outcome in the majority of bilateral cases, and many unilateral ones, at an early age.

This, in turn, is supported by the analysis of 200 cases of urinary infection in children.

Differentiation is suggested as a practical measure between this form of chronic pyelonephritis (atrophic pyelonephritis) and that which occurs at a later age-in which scar formation is less evident-as the natural histories of the two types appear to differ widely.

Our grateful thanks are due to colleagues both at University College Hospital and the Queen Elizabeth Hospital for Children who have allowed us to study their cases, and the unfailing and enthusiastic support of the radiographers and nurses at both hospitals. We are particularly indebted to Dr. E. McIlrath for his great help with case analysis, and to Drs. J. M. Smellie, D. Edwards, and I. C. S. Normand for allowing me to quote from our combined article.

\section{REFERENCES}

Campbell, M. (1951). Clinical Pediatric Urology. Saunders, Philadelphia. Escherich, quoted by Trump, J. (1897). Ueber Colicystitis in Kindesalter. fb. Kinderheilk., N.F., 44, 268.

Hodson, C. J. (1959). Proc. roy. Soc. Med., $52,669$.

Hodson, C. J. (1959). Proc. roy. Soc. Medicine (Balion C. R., Hewitt

Kunin, C. M., Deutscher, R., and Paquin, A. (1964). Ibid., 43, 91. Smellie, J. M., Hodson, C. J., Edwards, D., and Normand, I. C. S. (1964). Brit. med. F., 2, 1222 .

Smith, J. F. (1962). F. clin. Path., 15, 522

Weiss, S., and Parker, F. (1939).'Medicine (Baltimore), 18, 221. 